Relations industrielles

Industrial Relations

\title{
Organisations ouvrières au Canada, 48e edition, 1959, publié par la Direction de l'économique et de la recherche, Ministère du Travail, Canada, Ottawa, 96 pp.
}

\section{Gérard Dion}

Volume 15, numéro 4, octobre 1960

URI : https://id.erudit.org/iderudit/1021951ar

DOI : https://doi.org/10.7202/1021951ar

Aller au sommaire du numéro

Éditeur(s)

Département des relations industrielles de l’Université Laval

ISSN

0034-379X (imprimé)

1703-8138 (numérique)

Découvrir la revue

Citer ce compte rendu

Dion, G. (1960). Compte rendu de [Organisations ouvrières au Canada, 48e edition, 1959, publié par la Direction de l'économique et de la recherche,

Ministère du Travail, Canada, Ottawa, 96 pp.] Relations industrielles / Industrial

Relations, 15(4), 514-514. https://doi.org/10.7202/1021951ar

Tous droits réservés (C Département des relations industrielles de l’Université Laval, 1960
Ce document est protégé par la loi sur le droit d'auteur. L'utilisation des services d'Érudit (y compris la reproduction) est assujettie à sa politique d'utilisation que vous pouvez consulter en ligne.

https://apropos.erudit.org/fr/usagers/politique-dutilisation/ 
previously done. It also seems very odd that these « beliefs » and the impossibility of giving the times requested disappear after discharges and he undertook to furnish the required data. It was admitted in evidence that all times which he had previously reported were approximate and it was made abundantly clear that this was the request in this instance. The Board does find that there was insubordination and then proceeds to substitute their judgment for that of Management disregarding the fact that they have no such mandate.

There has been no violation or misinterpretation of any clause and there is no authority for this Board to determine or alter any penalty, therefore, the Board has exceeded its jurisdiction.

\section{RECENSIONS - BOOK REVIEWS}

Organisations ouvrières au Canada, 48e édition, 1959, publié par la Direction de l'économique et de la recherche, Ministère du Travail, Canada, Ottawa, 96 pp.

C'est le nom sous lequel désormais se présente la publication annuelle que nous connaissions sous le titre «Syndicalisme ouvrier au Canada ».

La raison de ce changement est que le répertoire des groupements ouvriers ne se borne plus aux syndicats rattachés à des centrales, mais comprend pour la première fois les organisations locales indépendantes de plus de cinquante membres, qui ont été certifiées comme agent négociateur en vertu de la loi des relations ouvrières appropriée. Pour éviter des susceptibilités, on prend la peine de noter: \& Le fait qu'une organisation paraît dans cette brochure n'implique aucune reconnaissance officielle. Les critères déterminant l'inclusion des organisations dans cette publication n'ont été fixés que dans le but de faciliter l'application de méthodes statistiques cohérentes ».

Comme nos lecteurs connaissent déjà par les éditions antérieures l'utilité de tous les renseignements fournis et l'excellence de cette publication, il nous suffira de remarquer certains changements que nous regrettons. Ainsi, les données statistiques sont moins complètes que par le passé. On ne trouve plus la répartition des unions locales et effectifs par industrie, ni par zône du mar- ché du travail ni par province. De même, dans le répertoire des organisations, ne sont pas indiquées, comme autrefois, les localités où elles possèdent des unités, mais seulement la répartition provinciale. Peut-être, a-t-on des raisons particulières pour n'avoir point fourni ces renseignements, mais c'est dommage. Faut-il aussi souligner que cette édition française nous parvient avec une année de retard?

\section{Gérard Dion}

Management's Right to Manage, by George W. Torrence. A BNA Operations Manual, The Bureau of National Affairs, Inc., Washington 7, D.C., $1959,109 \mathrm{pp}$.

Le but de cette étude est d'examiner ce qui est advenu du droit de gérance que possèdent les dirigeants des entreprises. L'auteur s'adresse aux employeurs afin de leur permettre d'évaluer leur position.

L'ouvrage est divisé en six parties: de quel droit il est question; importance du droit de gérance; ce qui est advenu du droit de gérance, en général; ce qui est advenu du droit de gérance dans des cas particuliers; comment se perd le droit de gérance; comment en arriver à préserver le droit de gérance.

Dans son travail, l'auteur laisse delibérément de côté les restrictions au droit de gérance provenant de la loi ou de la règlementation gouvernementale pour se confiner à celles qui arrivent dans la pra- 